\title{
Development of an imperialist competitive algorithm (ICA)-based committee machine to predict bit penetration rate in oil wells of Iran
}

\author{
Ayub Abbasi Garavand ${ }^{1}$, Dr. Gholamreza Esmaeilian ${ }^{1}$ \\ ${ }^{1}$ Department of industrial engineering, Payammoor tehran university, tehran, iran
}

\begin{abstract}
Drilling operation of a well is one the most expensive and time consuming procedures of oil and gas exploitation. Oil companies are always seeking for safe and cost-effective techniques for drilling. The main goal and motivation of drilling optimization is achieving the highest efficiency of work. Optimization and minimization of operational costs is one of the most important prerequisites of any engineering project. Rate of penetration is a crucial factor $\mathrm{n}$ drilling controlling cost and time of drilling. In the current research, capabilities of single independent intelligent models are employed for developing a hybrid committee machine that can predict bit penetration bit with high accuracy. To get this goal, three single intelligent models, including neural network, fuzzy logic and neuro-fuzzy, are trained. In the second step, the outputs of these models are integrated by imperialist competitive algorithm (ICA). Finally, a linear equation is achieved which gets outputs of single models as inputs and integrate them somehow the final results is closer to the actual value. The developed ICA-based committee machine is tested by 145 real data points gathered from the drilled wells in an oil field. Correlation of actual and predicted value of ROP obtained from committee machine shows that the model predicts ROP with accuracy of 88 percent. Such model can be used for optimization of drilling parameters in future drilling operations.
\end{abstract}

Keywords: Bit rate of penetration; Committee machine; Imperialist competitive algorithm; Decreasing operational costs of drilling 


\section{Introduction}

Hydrocarbon reserves were usually trapped in deep underground formations; Due to this fact, drilling a well is a costly operation for oil companies. Optimizing time and cost of drilling is the main goal of companies for saving money in spite of reaching the hydrocarbon. Indeed, many parameters control the drilling operation, among them three parameters should be noticed including weight on bit (WOB), rotation of the bit (rotation per minute (RPM)) and drilling mud flow. Determining the optimal rate of drilling, rate of penetration (ROP) has been always one of the main concepts of drilling engineering. Generally, two categories of parameters are affecting the rate of penetration. First category includes controllable and the second are uncontrollable parameters, also known as environmental. Variation of controllable parameters is more usual compared to the environmental ones since management of earth behaviors has been difficult and sometimes impossible. Interaction of these parameters with each other makes it difficult to develop a comprehensive model for studying ROP. For example, rate of penetration is considerably affected by formation properties. Mineralogy, porosity, permeability, density and strength are some of properties that control penetration rate. Bit design and nozzle arrangement are two parameters that change penetration rate while their role is somehow unknown. Due to high uncertainty in modeling of ROP, it is necessary to consider several factors of drilling data to develop an applicable model. The complexity of this task has motivated researchers to continuously work on ROP models to close synthetic models to reality as much as possible.

\section{Literature review}

All researches in regard to ROP can be divided into two points of view. First, conventional methods which were mainly used 30 years ago and they were based on mathematical concepts and theories. Galle and Woods (1960) are the pioneers of ROP optimization. Their research studied the relation of drilling parameters and ROP for the first time. However, they only considered WOB and RPM as the model input for developing an analytical function.Maurer
(1962) developed a ROP model for roller cone bits; however, the model did not present an acceptable performance in reality. Mechem and Fullerton (1965) developed a relationship in which ROP can be determined by formation drillability WOB, RPM, well depth, pressure and hydraulic condition of drilling mud. Lummus (1970) introduced the concept of optimization in drilling and effect of various parameters on drilling performance. In his method the necessary information were gathered from different sources including logging data (in special sonic log), recorded data of bit, drilling mud data, drill pipe torque, pump pressure, drilling rate, hole size and well depth. Gue (1988) presented a theoretical analysis of function of cost versus ROP. This analysis provided a simple method for searching the best parameters of drilling. Wojtanowicz and Kuru (1993) presented a novel method for drilling optimization by roller cone bits. Their method was based on a two-step dynamic programming and could be used locally for each bit and totally for the whole well. Barragan et al. (1997) developed a novel model which optimized ROP through Monte Carlo Simulation. Advancement of computers made it possible to study drilling data more accurate and develop comprehensive models. Intelligent models such as neural networks and genetic algorithm are some the results of computer advancements. Such models have been used by hundreds of researches in the world of engineering and their capability was proved by their accurate performance in risk minimization and cost optimization of engineering projects. Application of intelligent models has not limited to engineering world but also biologists and medical scientists have benefited from their advantage. Military is another branch of intelligent models application. Petroleum engineers have used intelligent systems for cost reduction and time optimization in drilling operations. Karri (1999) developed a model for prediction of drill pipe torque using neural network models and drilling data. Karri and Kiatcharoenpol (2003) employed neural network models to present a model for determination of effective usage of bottom-hole assembly. Rudolf (2006) used intelligent models to predict hydraulic characteristics of drilling operation such as pump pressures. Bahari et al. (2008) tried to develop a formula to predict ROP from the 
coefficients of Bourgoyne and Young model through genetic algorithm, which is one of the intelligent optimization techniques inspired from the Darwinian principle of evolution theory. Recently, SafaeeArdekani and Shadizadeh (2013) used both of neural network and linear regression approaches for development of drilling trip time model. They concluded that intelligent network model performed better compared to linear regression. Basarir et al. (2014) employed adaptive neuro-fuzzy model to predict the penetration rate of diamond bit. Monazami et al. (2012) used artificial neural network for prediction of ROP in one of Iranian oil fields. AlArfaj et al. (2012) presented an application of computational intelligent models in prediction of ROP. Bhatnagar and Khandelwal (2012) evaluated the drilling performance using an intelligent approach. Jacinto (2013) developed a model for prediction of ROP in salty formations of Brazil by a Bayesian clustering network. Duan et al. (2015) employed the advantages of an optimization algorithm for designing a neural network model to predict ROP. Studying the previous literatures shows that single independent intelligent models have been widely used for ROP prediction. In the current research a hybrid intelligent committee machine presented which integrates three singular expert systems. Imperialist competitive algorithm reaps the advantages of each model and covers the drawbacks of models with a model that performed better. In the next section, basic concepts of the proposed methodology are discussed.

\section{Methodology}

Intelligent models and novel techniques of data management are two factors for solving engineering problems. In the currents research an integrated intelligent model is developed in two steps. First, three singular models including neural network, fuzzy logic and neuro-fuzzy, are developed independently. Then, the outputs of these models are integrated by an imperialist competitive algorithm (ICA). Here, the fundamental of these models are explained below.

\section{3-1- Neural network model}

Artificial neural networks are intelligent models inspired from the natural intelligence of human brain. Now days, application of neural networks is widely extended in oil and gas industry. An artificial neural network composes of three main layers named input, hidden and output layer. Each layer contains some neurons. Number of neurons in input and output layer is equal to number of inputs and outputs while complexity and structure of training data determine number of hidden layer neurons. Transfer functions are the main parts of each neuron controlling model performance. Log-Sigmoid, Tan-Sigmoid and purelin are three well-known transfer functions. Fig. 1 shows a three layered neural network. A neural network model can be used for function estimation, curve fitting, pattern recognition and classification programs. Weights and biases of a network are tuned in training step somehow mean squared error of the model is minimal.

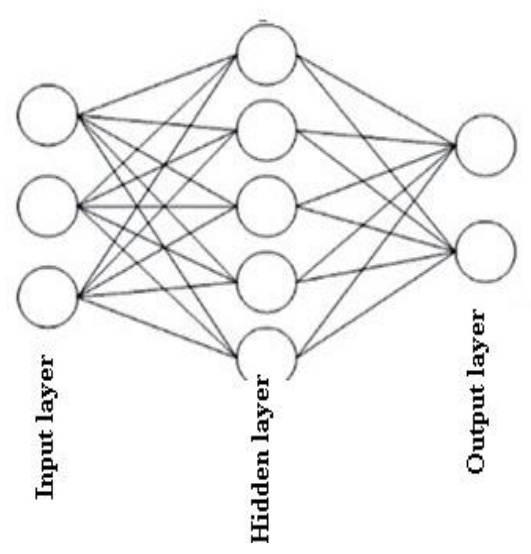

Fig. 1: Structure of a three layered artificial neural network

\section{3-2- $\quad$ Fuzzy logic}

Fuzzy logic (FL) theory, introduced by Zadeh in 1965, is an extension of traditional Boolean logic (0 and 1). In fuzzy set theory, the requirement of sharp boundaries of classical sets is abandoned and it is in general a matter of degree (Demico and Klir, 2004). Numbers, ranging from 0 to 1 , express the degree of membership in a fuzzy set as degree of truth. The ability of fuzzy sets to express gradual transitions from membership to nonmembership provides meaningful and powerful representation of uncertainties (Klir and Yuan, 1995). It is suggested to use fuzzy logic for determination of an acceptable solution for 
problems, which are convoyed, by vagueness and imperfection. The main part of a fuzzy set is fuzzy inference system (FIS), which is formulating the inputs to an output. Mamdani and Assilian (1975) introduced the first type of FIS and Takagi and Sugeno (1985) initiated the second one. Both of FISs, attempt to control a system by integrating a set of linguistic control rules while the main difference between methods is output membership function, which is constant for the Takagi-Sugeno fuzzy inference system (TS-FIS). In the TS-FIS, a clustering process defines membership functions. Each of the clusters refers to a membership function to generate the if-then rules. The fuzzy system makes a sum of all then parts and uses a defuzzification method to give the final output.

\section{3-3- Neuro-fuzzy model}

The main drawbacks of fuzzy inference systems are difficulty and uncertainty in selection and generating membership functions and "if-then" rules. Neuro-fuzzy model employed the advantages of neural network models for identification of fuzzy rules and tuning membership functions. In contrast whit normal fuzzy systems that can have several outputs, neuro-fuzzy models are single output models.

\section{3-4- Imperialist competitive algorithm}

Imperialist competitive algorithm is a novel optimization technique developed based on principals and rules of social and political evolutions of human society. Atashpaz-Gargari and Lucas (2007) introduced this model. Similar to all population based models, ICA needs an initial population which is defined as "countries". Each country includes $\mathrm{N}$ evolutionary vector. ICA uses these countries for determination of objective function also known as cost function. Countries with lowestcost are classified as imperialists ( $\mathrm{Nimp}$ ) while others are colonies $\left(\mathrm{N}_{\mathrm{col}}\right)$. In the next step, colonies are divided between the imperialists. A set of one imperialist and some colony is named empire. Power of each empire is determined based on number of its colonies. Higher number of colonies, higher power of the empire. Each colony moves toward its imperialist from various directions of optimization. This process is named assimilation. Although movement in direction of vector " $\mathrm{x"}$ increase chance of the colony to find the best solution; however, it also increases risk of sticking in local minimal. Therefore, it is necessary to enlarge searching space of the colony. This task is made possible by definition of a deviation angle $(\theta)$, which is a freedom of colony to move in different directions. Fig. 2 shows that the deviation angle as a random variable with uniform distribution which is defined the direction of the colony.

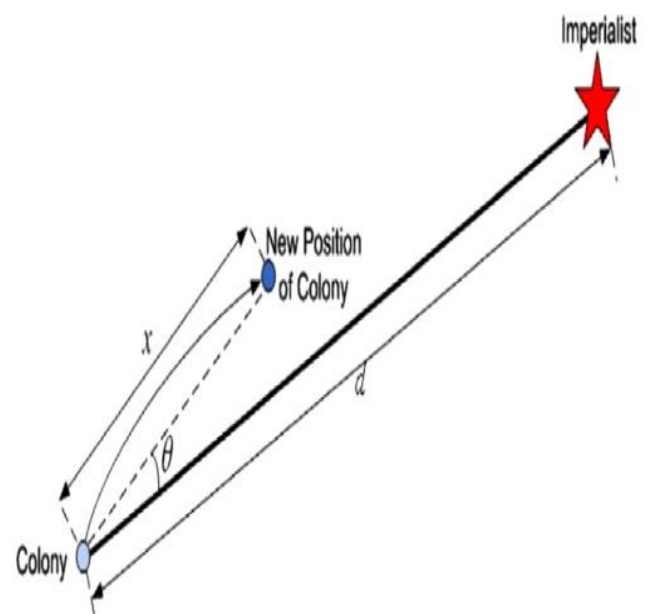

Fig. 2: Movement of the colony toward the imperialist whit deviation angle $(\theta)$

Another parameter known as revolution is also defined in ICA which is changed the colony's cost randomly. Revolution decreases the possibility of sticking the algorithm in local minima. Fig. 3 represents the process of happening a revolution in the algorithm.

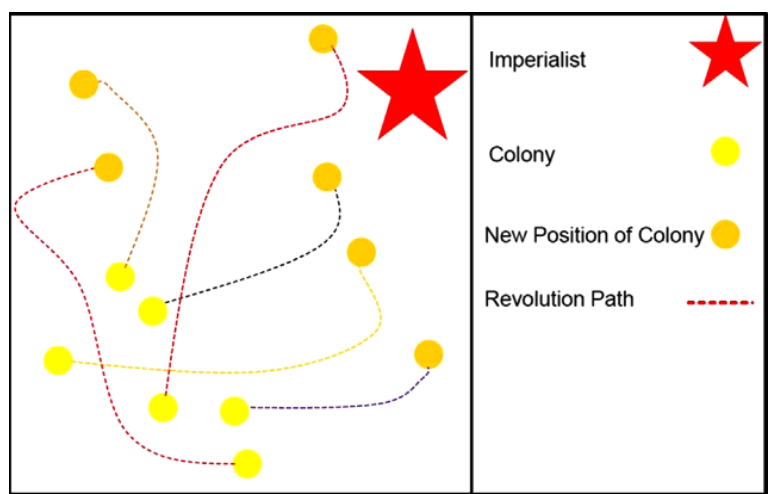

Fig. 3: A sudden change in objective function value of colonies

During the assimilation and revolution the cost value of colonies are changed. If the new 
position of the colony is lower than the empire's imperialists then the position of better colony is changed with the imperialists. In the next step, imperialistic power of each empire is determined by Eq.1 as shown below.

$C_{n}=\operatorname{Cost}\left(\right.$ imperialist $\left._{n}\right)+$

E. mean (Cost (Colony of empire $\left.{ }_{n}\right)$ ) (Eq: 1 )

Where $\varepsilon$ is positive small number tuned by the algorithm operator. Based on the total cost of empires, imperialistic competitions take place and all empires try to take the possession of colonies of other empires and control them. The imperialistic competition gradually brings about a decrease in the power of weaker empires and an increase in the power of more powerful ones. This imperialistic challenge is approached by just selecting some (usually one) of the weakest colonies of the weakest empire and making a competition among all empires to possess these (this) colonies. This process will be continued and causes the countries to converge to the global minimum of the cost function. At the end all the empires except the most powerful one will be collapsed and the colonies will be handled by this unique empire. It should be noted that imperialist and colonies have the same position and power in this stage (Atashpaz-Gargari and Lucas, 2007). In this step, the objective function is converged to its minimal point, where cost value of all colonies and the final imperialist is equal.

\section{3-5- Principal of committee machine}

According to the principle of divide and conquer, a complex computational task is solved by dividing it into a number of computationally simple tasks and then combining the solutions to those tasks. A CM consists of a group of intelligent systems, which combines the outputs of each system and thus reaps the benefits of all work, with little additional computation. The experts can be empirical formulas, neural networks, a decision tree or another type of algorithm (Sharkey, 1996). The idea behind the committee machine is to fuse the knowledge acquired by experts in order to arrive at an overall decision that is superior to that of any of the individual experts acting alone (Haykin, 1991). Fig. 4 shows the flowchart of an intelligent committee machine developed based on three singular intelligent models.

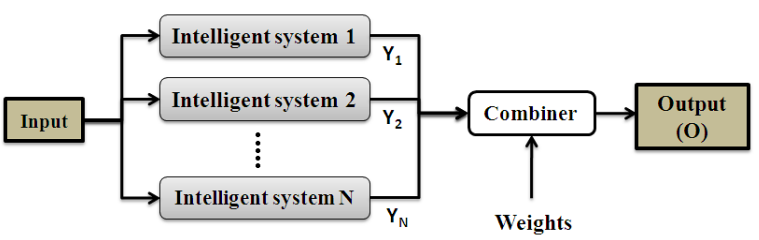

Fig. 4: A committee machine flowchart

\section{4- Data gathering and preparation}

To prepare the necessary data for the current article, 822 data points of drilling data from the drilled well of Iran are gathered. Among the available data points, 737 ones are used as training and remain used for model verification. First, the most effective drilling parameters on ROP are distinguished. Mechanism of bit penetration shows that drilling mud characteristics, drill pipe torque, mud pressure, amount of injected mud (GPM: gallon per minute), weight on bit (WOB), rotation speed of bit (RPM: rotation per minute) have considerably impact on ROP. These parameters should be tuned so that ROP stands at it optimum point during drilling; otherwise, not only time drilling increases but also bit wear and stocking risk of bit in underground formations is increased.

\section{5- Conclusions and results}

In the present article, the capabilities of single intelligent models are employed for development of a hybrid intelligent committee machine to predict bit penetration rate based on online drilling data. Three single intelligent model, including neural network, fuzzy logic and neuro-fuzzy, are integrated through an optimization algorithm named imperialist competitive algorithm (ICA).

\section{5-2- Designing the neural network model}

Neural network is the first component of the committee machine model, which is explained in this section. A feed forward back propagation algorithm is selected for the NN model. Transfer functions of input layer to hidden layer and hidden layer to output layer are "tansig" and "purelin", respectively. A levenberg-Marquardt 
algorithm is selected as the training algorithm of the model. This algorithm has an accurate performance in curve fitting and regression problems. Convergence in short time is another advantage of the algorithm. Number of neurons in hidden layer is determined in a try-and-error procedure, which is shown that 9 neurons is the optimum value. Number of epochs is tuned at 1000. The results show that convergence time of the NN model is less than 20 seconds.

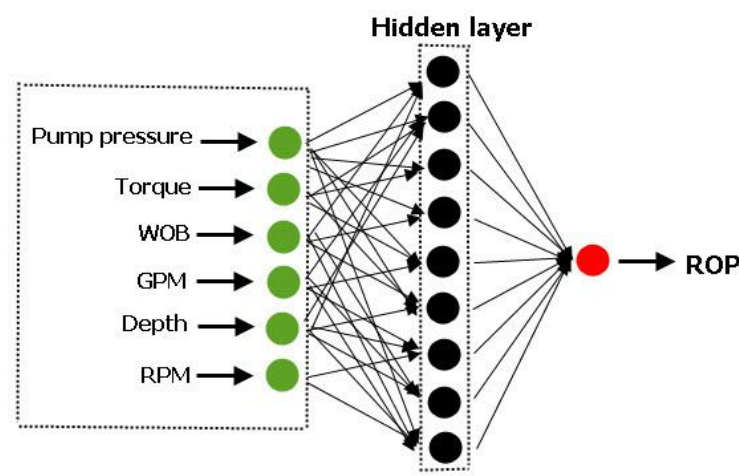

Fig. 5: Schematic illustration of the structure of the designed neural network model (Weight on bit, amount of injected mud, rotation speed of bit are shown by WOB, GPM, RPM, respectively)

This time can be tolerate regarding the number of training data and the complexity of inputs and desired output relationship. Mean square error (MSE) of the model for training and testing data is 2.37 and 3.05, respectively. Correlation coefficient of predicted values and desired output for training and testing data is 0.87 and 0.82 , respectively. Fig. 5 represents a schematic illustration of the designed model and relation of three layers of neural network.

\section{5-3- Designing fuzzy logic model}

Fuzzy logic model is the second component of the committee machine. A Takagi-Sugeno fuzzy inference system(TS-FIS) is employed. Subtractive clustering is used for determination of type and amount of membership functions of inputs and the desired output. The main parameter of the subtractive clustering affecting the number of fuzzy if-then rules and the accuracy of a fuzzy model is setting an optimal clustering radius. The optimal clustering radius was searched by performing the clustering process several times and gradually increasing the clustering radius from $0 \quad t \quad o 1$ (with0.1intervals).Thus, 10 fuzzy models with the different number of fuzzy if-then rules were established. Then, the fuzzy model with the highest overall accuracy was selected as the optimal model.The results are represented in table 1.

Table 1: radius of clustering, number of "if-then rules" and MSE of the fuzzy model

\begin{tabular}{|l|l|l|l|}
\hline No. & Clustering radious & No. "if-then" rules & MSE \\
\hline $\mathbf{1}$ & 0.1 & 450 & 351400 \\
\hline $\mathbf{2}$ & 0.2 & 41 & 50.17 \\
\hline $\mathbf{3}$ & 0.3 & 17 & 14.79 \\
\hline $\mathbf{4}$ & 0.4 & 10 & 6.39 \\
\hline $\mathbf{5}$ & 0.5 & 7 & 2.96 \\
\hline $\mathbf{6}$ & 0.6 & 5 & 3.77 \\
\hline $\mathbf{7}$ & 0.7 & 4 & 4.72 \\
\hline $\mathbf{8}$ & 0.8 & 3 & 7.09 \\
\hline $\mathbf{9}$ & 0.9 & 2 & 10.93 \\
\hline $\mathbf{1 0}$ & 1 & 2 & 11.05 \\
\hline
\end{tabular}

As indicated in table 1, 0.6 is the optimal value of clustering radius since the MSE reaches to its minimal point. The fuzzy model achieves an acceptable recognition of the relationship of inputs and the output in this value of clustering radius. Membership functions are Gaussian type, which are defined by the below equation. 
$\mathrm{f}(\mathrm{x}, \mu, \sigma)=\frac{\mathrm{e}^{-(\mathrm{x}-\mu)^{2}} / 2 \sigma^{2}}{\sigma \sqrt{2 \pi}}$
Where $\sigma$ and $\mu$ are the mean and standard deviation of data, respectively. Values of these parameters for designed membership functions is shown in table 2

(Eq. 2)

Table 2: Mean and standard deviation of membership functions parameters

\begin{tabular}{|c|c|c|c|c|c|c|}
\hline Inputs & \multicolumn{2}{|c|}{ Torque } & \multicolumn{2}{|c|}{ Injected mud } & \multicolumn{2}{|c|}{ WOB } \\
\hline Parameters & $\sigma$ & $\mu$ & $\sigma$ & $\mu$ & $\sigma$ & $\mu$ \\
\hline Mf1 & 2231 & 2100 & 201 & 1047 & 4.6 & 6 \\
\hline Mf2 & 2231 & 4400 & 201 & 1136 & 4.6 & 11 \\
\hline Mf3 & 2231 & 4933 & 201 & 1211 & 4.6 & 13 \\
\hline Mf4 & 2231 & 5100 & 201 & 1266 & 4.6 & 15 \\
\hline Mf5 & 2231 & 5310 & 201 & 1318 & 4.6 & 17 \\
\hline Inputs & \multicolumn{2}{|c|}{ Pump pressure } & \multicolumn{2}{|c|}{ RPM } & \multicolumn{2}{|c|}{ depth } \\
\hline parameters & $\sigma$ & $\mu$ & $\sigma$ & $\mu$ & $\sigma$ & $\mu$ \\
\hline Mf1 & 26 & 179 & 20 & 70 & 188 & 331 \\
\hline Mf2 & 26 & 202 & 20 & 89 & 188 & 526 \\
\hline Mf3 & 26 & 206 & 20 & 94 & 188 & 592 \\
\hline Mf4 & 26 & 220 & 20 & 128 & 188 & 718 \\
\hline Mf5 & 26 & 224 & 20 & 137 & 188 & 857 \\
\hline
\end{tabular}

Number of membership functions is equal to number of "if-then" rules. Fig. 6 shows the variation of membership functions of the fuzzy model, which are named as "mf1", "mf2", "mf3", "mf4" and "mf5".
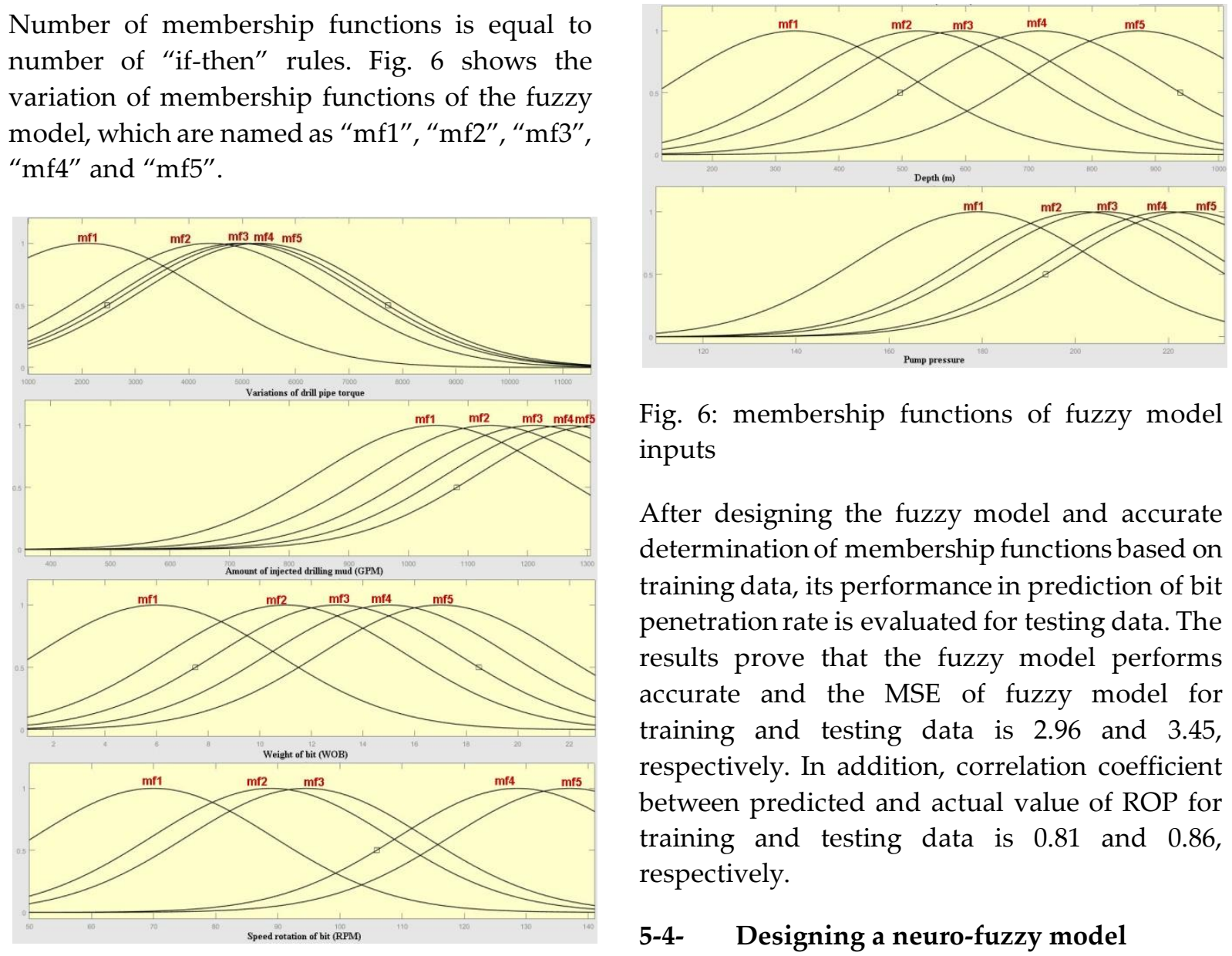

Fig. 6: membership functions of fuzzy model inputs

After designing the fuzzy model and accurate determination of membership functions based on training data, its performance in prediction of bit penetration rate is evaluated for testing data. The results prove that the fuzzy model performs accurate and the MSE of fuzzy model for training and testing data is 2.96 and 3.45, respectively. In addition, correlation coefficient between predicted and actual value of ROP for training and testing data is 0.81 and 0.86 , respectively.

\section{5-4- Designing a neuro-fuzzy model}

Neuro-fuzzy system is a powerful intelligent tool that integrates the advantages of both artificial 
neural network and fuzzy logic. In the structure of a NF system, a FL model enhances generalization capability of a NN system by providing more reliable output when extrapolation is needed beyond the limits of the training data. A hybrid optimization method is considered to train the membership functions. The hybrid optimization method is a combination of the least squares and backpropagation gradient descent methods. The number of epochs and error tolerance were set to 100 and 0 , respectively. After 26 epochs, no improvement was found in the performance of the model and the training was stopped. The effectiveness of the NF model relies on the optimal clustering radius. As with the fuzzy model, the initial model for the adaptive NF inference system was generated by applying a subtractive clustering on data. After introducing the training data the membership functions were adjusted and the MSE for training and testing data is determined 2.9 and 3.47, respectively. Correlation coefficient between predicted and actual value of ROP for training and testing data is 0.81 and 0.86 , respectively.

\section{5-5- Designing the ICA-based committee machine}

Committee machine is a hybrid model, which is used the advantage of an optimization algorithm for integrating the outputs of some single intelligent models. The integrated models improve the prediction accuracy by covering the drawbacks of each model with advantage of another model. In fact, the optimization algorithm determines the contribution of each model in the final integrated model somehow the results of the integrated model are closer to the actual values. Higher contribution of a model in the committee machine proves higher accuracy of that model when it was trained independently.

Optimization algorithms try to minimize the MSE of engineering problems. In the current research, the objective function of ICA is defined as a linear equation of three single models. The defined equation is shown below.

MSE $=\frac{1}{\mathrm{n}} \sum_{\mathrm{i}=1}^{\mathrm{n}}\left(\mathrm{W}_{1} \times \mathrm{NN}_{\mathrm{i}}+\mathrm{W}_{2} \times \mathrm{FL}_{\mathrm{i}}+\mathrm{W}_{3} \times \mathrm{NF}_{\mathrm{i}}-\right.$ ROP $)^{2} \quad$ (Eq. 3)
Where $\mathrm{W}_{1}, \mathrm{~W}_{2}$ and $\mathrm{W}_{3}$ are contribution of single intelligent models. This equation is introduced to ICA as a function while its variables are obtained outputs of single models and desired ROP. Number of initial countries of ICA is 80 . Number of primary imperialists is 8 ; therefore number of initial colonies will be 72 . Number of decades and rate of revolution is adjusted at 30 a 0.3, respectively. Increasing the amount of these numbers does not improve the accuracy of ICA whereas it increases convergence time of algorithm. Fig. 7 shows the performance of ICA for each decade and the final error in the last decade.

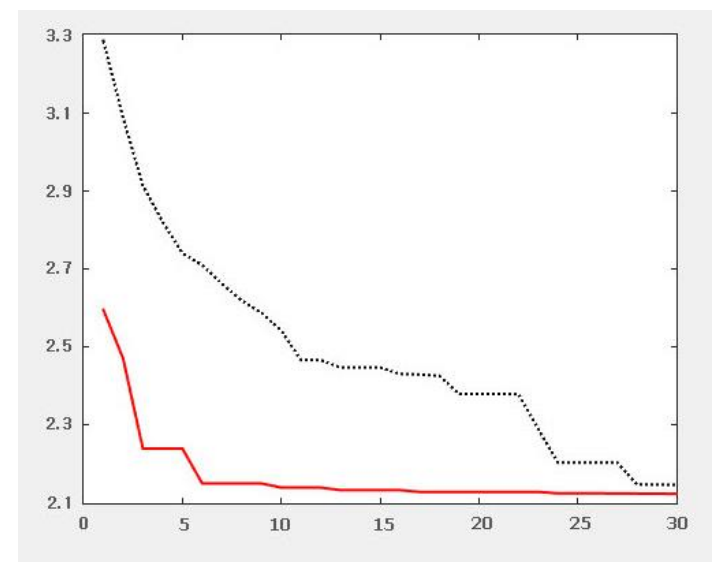

Fig. 7: MSE of ICA in 30 decade of convergence

The red and dotted line represent amount of objective function in the imperialists and the colonies, respectively. In the last decades of algorithms MSE of imperialists and the colonies converges to a same value. In this figure, after decade 28 there is no considerable difference between the MSE values. After running the ICA, weights of neural network, fuzzy model and neuro-fuzzy model adjusted at $0.81,0.15$ and 0.03 , respectively. These weights indicate that neural network model performed better in 81 percent of data points compared to two other models. The optimized weight coefficients were applied to Eq. 3 and the final output was calculated as belowEq. 4 .

$\mathrm{ROP}=0.81 \times \mathrm{NN}_{\mathrm{i}}+0.15 \times \mathrm{FL}_{\mathrm{i}}+0.03 \times \mathrm{NF}_{\mathrm{i}}$ (Eq. 4)

This equation is used for determination of ROP in training and testing data. The results show that MSE of training and testing data is 2.12 and 
2.51, respectively, which correspond to the $\mathrm{R}^{2}$ values of 0.89 and 0.88 , respectively.Both of MSE and $R^{2}$ values were improved compared to single independent models.
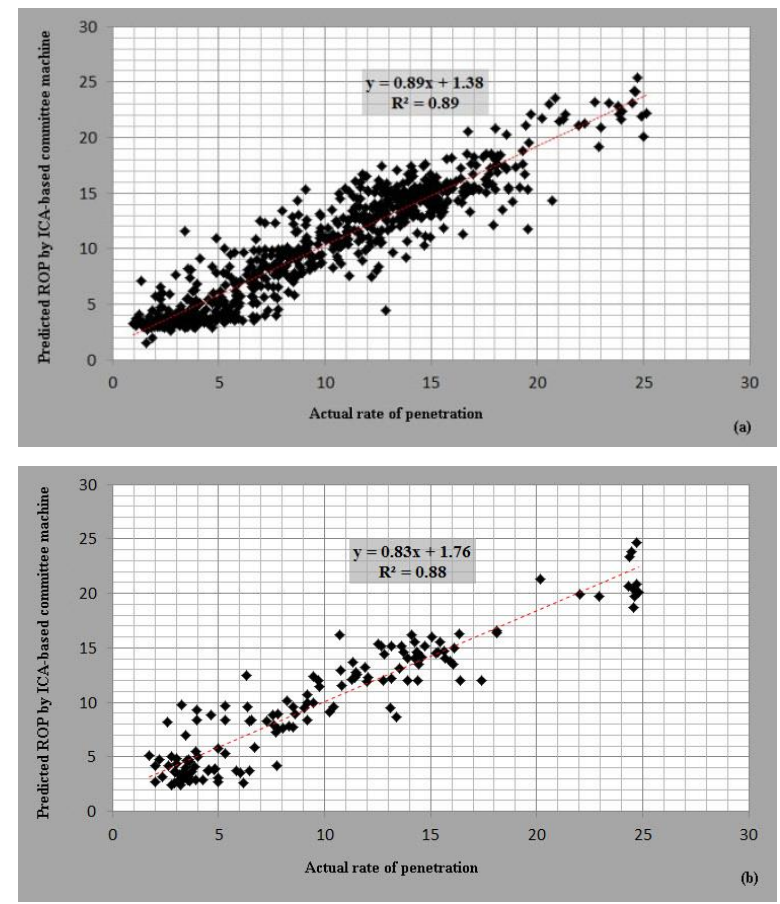

Fig. 8: Correlation coefficient between actual and predicted ROP by ICA-based committee machine for (a) training data and (b) testing data.

\section{6- Conclusion}

In the present research, an intelligent hybrid ICA-based committee machine was developed for prediction of ROP of drilling bit. This model can be used for optimization of drilling feature such as weight on bit, rotation speed, pump pressure and etc. Each of these parameters has direct and considerable impact on drilling costs. Table 3 ranks MSE values of single intelligent models and developed committee machine.

Table 3: comparing the performance of single and integrated intelligent models

\begin{tabular}{|r|r|r|r|r|}
\hline $\begin{array}{r}\text { Intelligent } \\
\text { model }\end{array}$ & \multicolumn{2}{|r|}{ MSE of prediction } & \multicolumn{2}{r|}{ Rank } \\
\cline { 2 - 5 } & Train & Test & Train & TEST \\
\hline $\begin{array}{r}\text { Neural } \\
\text { network }\end{array}$ & 2.37 & 3.05 & 2 & 2 \\
\hline $\begin{array}{r}\text { Fuzzy } \\
\text { model }\end{array}$ & 2.96 & 3.45 & 4 & 3 \\
\hline $\begin{array}{r}\text { Neuro- } \\
\text { fuzzy }\end{array}$ & 2.90 & 3.47 & 3 & 4 \\
\hline $\begin{array}{r}\text { Committee } \\
\text { machine }\end{array}$ & 2.12 & 2.51 & 1 & 1 \\
\hline
\end{tabular}

\section{References}

AlArfaj, Ibrahim, Amar Khoukhi, and Tuna Eren. "Application of Advanced Computational Intelligence to Rate of Penetration Prediction." Computer Modeling and Simulation (EMS), Sixth UKSim/AMSS European Symposium on. IEEE, 2012.

Basarir, H., L. Tutluoglu, and C. Karpuz. "Penetration rate prediction for diamond bit drilling by adaptive neuro-fuzzy inference system and multiple regressions." Engineering Geology 173: 1-9, (2014).

Bhatnagar, A., and Manoj Khandelwal. "An intelligent approach to evaluate drilling performance." Neural Computing and Applications 21.4: 763-770. (2012).

Bourgoyne, A.T., Jr. and Young, F.S., Jr.: "A Multiple Regression Approach to Optimal Drilling and Abnormal Pressure Detectiony", SPE 4238, SPE-AIME Sixth Conference on Drilling and Rock Mechanics, Austin, TX, January 1973.

Brett,F.J. and Millheim,K.K.:" The Drilling Performance Curve: A Yardstick for Judging Drilling Performance", 61st Annual Technical Conference and Exhibition of the Society of Petroleum Engineers,NewOrfeans, LA, October 5-8, 1986.

Bonet, L., Cunha, J.C.S. and Prado, M.G.: "Drilling Optimization: A New Approach to Optimize Drilling Parameters and Improve Drilling Efficiency", Drilling Technology, ASME, 1995.

Barragan, R.V., Santos, O.L.A. and Maidla, E.E.: "Optimization of Multiple Bit Runs", SPE 37644, 1997 SPE/IADC Drilling Conference, Amsterdam, The Netherlands, March 1997.

Jacinto C., FreitasFilho P.J., Nassar S.M., Rosenberg M., Rodrigues D.G., Lima M.D.C., 2013, Optimization models and prediction of drilling rate (rop) for the brazilian pre-salt layer, Chemical Engineering Transactions, 33, 823-828 DOI: 10.3303/CET1333138

Galle, E.M. and Woods, H.B.: "Best Constant Weight and Rotary Speed for Rotary Rock Bits", API Drilling and Production Practice, 1960. 
Guo,X.Z.:"A Preliminary Investigation on the Objective Function of Penetration Cost Applied to Optimized Drilling", International Meeting on Petroleum Engineering, Beijing,China,March 17-20,1988.

Karri, V., "RBF Neural Networks For Thrust and Torque Predictions in Drilling Operations", Proc. International Conference on Computational Intelligence and Multimedia Applications (ICCIMA), Sep. 1999, New Delhi, India, pp. 55-60.

Karri, V., Kiatcharoenpol, T., Tool Condition Monitoring in Drilling Using Artificial Neural Networks.Lecture Notes in Computer Science Volume 2903, 2003, pp 293-301.

Maurer, W.C.: "The 'Perfect-Cleaning' Theory of Rotary Drilling", Journal of Petroleum Technology, November, 1962.

Mechem,O.E. and Fullerton, H.B.Jr.,:"Computers invade the rig floor" Oil and Gas Journal, p14, 1965.

Miska, S. and Ziaja, M..B:"Mathematical Model of the Diamond- Bit Drilling Process and Its Practical Application", Annual Fall Meeting of the Society of Petroleum Engineers of AIME, December,1982.

Moradi, H., Bahari, M.H., Naghibi, M.B. and Bahari, A., Drilling rate prediction using an innovative soft computing approach. Scientific Research and Essays Vol. 5(13), pp. 1583-1588, 4 July, 2010.

Monazami, M., Hashemi, A., Shahbazian, M., DRILLING RATE OF PENE TRATION PREDICTION USING ARTIFICIAL NEURAL NETWORK: A CASE STUDY OF ONE OF IRANIAN SOUTHERN OIL FIELDS. Journal of Oil and gas Business. No 6, 21-31. (2012).

$$
\begin{array}{ccc}
\text { Lummus, J. } & \text { L.:"Drilling } & \text { Optimization", } \\
\text { JOURNAL } & \text { OF } & \text { PETROLEUM } \\
\text { TECHNOLOGY,p1379, NOV } 1970 .
\end{array}
$$

SafaeeArdekani, O., Shadizadeh, S.R., "Development of drilling trip time model for southern Iranian oil fields: using artificial neural networks and multiple linear regression approaches", Journal of Petroleum Exploration and Production Technology, December 2013, Volume 3, Issue 4, pp 287295.
Atashpaz-Gargari, E., Lucas, C., 2007. Imperialist competitive algorithm: an algorithm for optimization inspired by imperialistic competition. In: Proceedings of the IEEE Congress on Evolutionary Computation, pp. 4661-4667

Jinan Duan, Jinhai Zhao, Li Xiao, Chuanshu Yang, Changsheng Li, 2015. A ROP Optimization Approach Based on Improved BP Neural Network PSO. Advances in Swarm and Computational IntelligenceLecture Notes in Computer Science Volume 9142, 2015, pp 11-18.

Wojtanowicz, A.K., and Kuru, E.: "MinimumCost Well Drilling Strategy Using Dynamic Programming", Joumal of Energy Resources Technology, Transactions of the ASME, December 1993.

Zadeh, L.A., 1965. Fuzzy sets. Information and Control 8, 338-353. 